\title{
Therapeutic Plasmapheresis in Primary Presentation of Diabetes Mellitus With Diabetic Ketoacidosis, Hypertriglyceridemia and Acute Pancreatitis
}

\author{
Sherleen Gandhamª, Farhanah Yousaf a, b, Yin Win ${ }^{a}$, Sheng Kuo ${ }^{a}$, Chaim Charytan ${ }^{\mathrm{a}}$, Bruce Spinowitz ${ }^{\mathrm{a}}$, \\ Marilyn Galler ${ }^{\mathrm{a}}$
}

\begin{abstract}
Severe hypertriglyceridemia (SHTG)-induced acute pancreatitis has been well described. Currently accepted therapeutic options are limited. We report a case of acute pancreatitis associated with hypertriglyceridemia and diabetic ketoacidosis that was safely and effectively managed with plasmapheresis. A 38-year-old female with history of gestational diabetes presented with acute onset of nausea and abdominal pain. She denied alcohol use and was on oral contraceptive pills. On physical exam, she was afebrile, normotensive, and tachycardic with mild abdominal distension and diffuse tenderness. Diagnostic tests revealed a serum glucose of $414 \mathrm{mg} / \mathrm{dL}$, triglycerides $>816 \mathrm{mg} /$ $\mathrm{dL}$, amylase of $106 \mathrm{U} / \mathrm{L}$, lipase of $272 \mathrm{U} / \mathrm{L}$, anion gap of 23, BUN of $13 \mathrm{mg} / \mathrm{dL}$, creatinine of $0.3 \mathrm{mg} / \mathrm{dL}$, sodium of $120 \mathrm{mmol} / \mathrm{L}, \mathrm{HCO}_{3}$ of $14 \mathrm{mmol} / \mathrm{L}$, and positive urine ketones. Computed tomography (CT) findings were consistent with severe acute pancreatitis. The patient was managed conservatively on day 1 . Due to persistence of symptoms, plasma exchange using NxStage plasmafilter was performed on day 2 and day 3, which resulted in significant reduction of the triglyceride level and resolution of abdominal pain. Patient was discharged home with gemfibrozil and glyburide as maintenance therapy. The exact mechanism of hypertriglyceridemia-induced pancreatitis is not clear. It has been postulated that hyperviscosity of blood due to lipid particles causes ischemia in the pancreas, releasing inflammatory mediators and leading to pancreatic necrosis and inflammation. The advantage of plasmapheresis over conservative management is the removal of lipid particles in a relatively short period of time and clearance of triglyceridemia-associated pro-inflammatory agents.
\end{abstract}

Keywords: Therapeutic plasmapheresis; Acute pancreatitis; Hypertriglyceridemia-induced pancreatitis; Severe hypertriglyceridemiaassociated pancreatitis; Diabetes mellitus; Diabetic ketoacidosis; Hypertriglyceridemia

\footnotetext{
Manuscript accepted for publication November 26, 2014

aDivision of Nephrology, New York Hospital Queens, Flushing, NY, USA ${ }^{\mathrm{b}}$ Corresponding Author: Farhanah Yousaf, New York Hospital Queens, 56-45 Main Street, Flushing, NY 11355, USA. Email: fay9005@nyp.org
}

doi: http://dx.doi.org/10.14740/wjnu189w

\section{Introduction}

Acute pancreatitis is a fatal condition which is associated with a mortality rate between $6 \%$ and $23 \%$ in the adult population [1-3]. In the United States, the incidence of acute pancreatitis has increased consistently to 49 per 100,000 persons from 1988 to 2004 [4]. Severe hypertriglyceridemia (SHTG) is the third most common cause of acute pancreatitis after alcohol and gallstones, and accounts for as many as $10 \%$ of all cases of acute pancreatitis [5-8]. Much of the etiology of acute hypertriglyceridemia-induced pancreatitis is not fully understood, including definitive threshold concentrations, genetic variations and environmental factors. Generally, triglyceride levels above $1,000 \mathrm{mg} / \mathrm{dL}$ are associated with acute pancreatitis. A recent retrospective review even suggested that triglyceride levels below $1,772 \mathrm{mg} / \mathrm{dL}$ are unlikely to be the primary cause of acute pancreatitis [9].

The currently accepted treatment options for acute hypertriglyceridemia-induced pancreatitis consist of nutritional intervention and anti-lipemic medications in addition to the current standard management of acute pancreatitis including risk stratification, aggressive hydration, maintenance of nil per os (NPO), and procedures such as endoscopic retrograde cholangiopancreatography (ERCP) and antibiotics if indicated [10]. Though effective, these therapies often take days to achieve clinical improvement. Therapeutic plasmapheresis may be a safe and effective alternative for the management of severe hypertriglyceridemia-associated pancreatitis (SHAP).

We report a case of primary presentation of diabetes mellitus in a patient with diabetic ketoacidosis, acute pancreatitis and hypertriglyceridemia, which was safely and effectively managed with therapeutic plasmapheresis.

\section{Case Report}

A 38 -year-old female with a body mass index of $20.2 \mathrm{~kg} / \mathrm{m}^{2}$ and past medical history of gestational diabetes presented to the Emergency Department of New York Hospital Queens complaining of nausea and abdominal pain of acute onset. She denied alcohol use and was taking only oral contraceptive pills at the time of presentation. On initial physical exam, she was afebrile, normotensive, and tachycardic, with mild abdominal distension and diffuse abdominal tenderness. Initial laboratory 


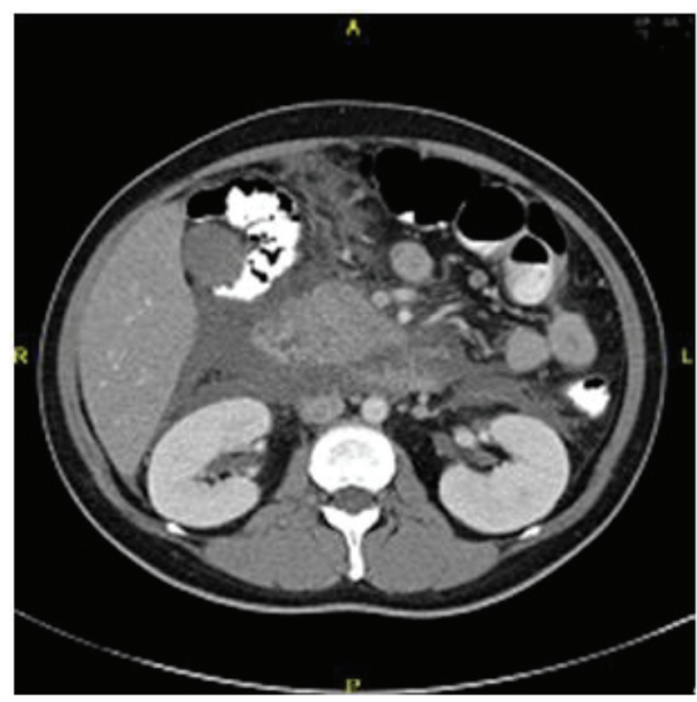

Figure 1. CT scan from day 1 of hospitalization showing diffusely inflamed pancreas.

tests revealed a serum glucose of $414 \mathrm{mg} / \mathrm{dL}$, cholesterol $>727$ $\mathrm{mg} / \mathrm{dL}$, triglycerides $>816 \mathrm{mg} / \mathrm{dL}$ (the exact levels of cholesterol and triglycerides were not measured by our laboratory), amylase of $106 \mathrm{U} / \mathrm{L}$, lipase of $272 \mathrm{U} / \mathrm{L}$, anion gap of 23, BUN of $13 \mathrm{mg} / \mathrm{dL}$, creatinine of $0.3 \mathrm{mg} / \mathrm{dL}$, sodium of $120 \mathrm{mmol} / \mathrm{L}$, $\mathrm{HCO}_{3}$ of $14 \mathrm{mmol} / \mathrm{L}, \mathrm{LDH}$ of $527 \mathrm{U} / \mathrm{L}, \mathrm{AST}$ and ALT $<5 \mathrm{U} / \mathrm{L}$, $\mathrm{WBC}$ of $10,540 / \mu \mathrm{L}$, negative $\mathrm{HCG}$, and urine positive for ke- tones. A spiral computed tomography (CT) scan of the abdomen and pelvis showed enlargement of the pancreas and extensive infiltration of the peripancreatic fat with a large amount of peripancreatic fluid. No evidence of abscess or pseudocyst was found. The liver showed signs of fatty infiltration without hepatic lesions or evidence of cholelithiasis. No adrenal masses were identified and the kidneys, abdominal aorta, uterus and bladder showed no abnormalities (Fig. 1). These clinical and radiological findings are consistent with a diagnosis of severe acute pancreatitis.

The patient was admitted and kept NPO. On admission, the patient had a Ranson score of 2 with a predicted mortality of $1 \%$. Aggressive hydration, gemfibrozil $600 \mathrm{mg}$ BID, insulin, heparin, and morphine were initiated on the first day of admission. These standard therapeutic interventions did not yield clinical improvement in the first $24 \mathrm{~h}$, so the decision to begin therapeutic plasmapheresis with NxStage ${ }^{\circledR}$ plasmafilter was made. A repeat triglyceride level within $24 \mathrm{~h}$ after the first session of plasmapheresis was $829 \mathrm{mg} / \mathrm{dL}$. At $48 \mathrm{~h}$, the patient had a predicted mortality risk of $16 \%$ based on a Ranson score of 4 which included glucose $>200 \mathrm{mg} / \mathrm{dL}, \mathrm{LDH}>350 \mathrm{IU} / \mathrm{L}$, calcium $<8 \mathrm{mg} / \mathrm{dL}$, and a base deficit $>4 \mathrm{mEq} / \mathrm{L}$.

A second session of plasmapheresis was performed on the third day of admission, which yielded a reduction in triglyceride levels to $380 \mathrm{mg} / \mathrm{dL}$. During the patient's clinical course, triglyceride levels increased to $605 \mathrm{mg} / \mathrm{dL}$ by day 5 without recurrence of symptoms, but steadily decreased thereafter to $380 \mathrm{mg} / \mathrm{dL}$ at time of hospital discharge (Fig. 2). A follow-up CT scan was performed on day 7 which demonstrated develop-

\section{Triglyceride Levels Obtained During Hospitalization}

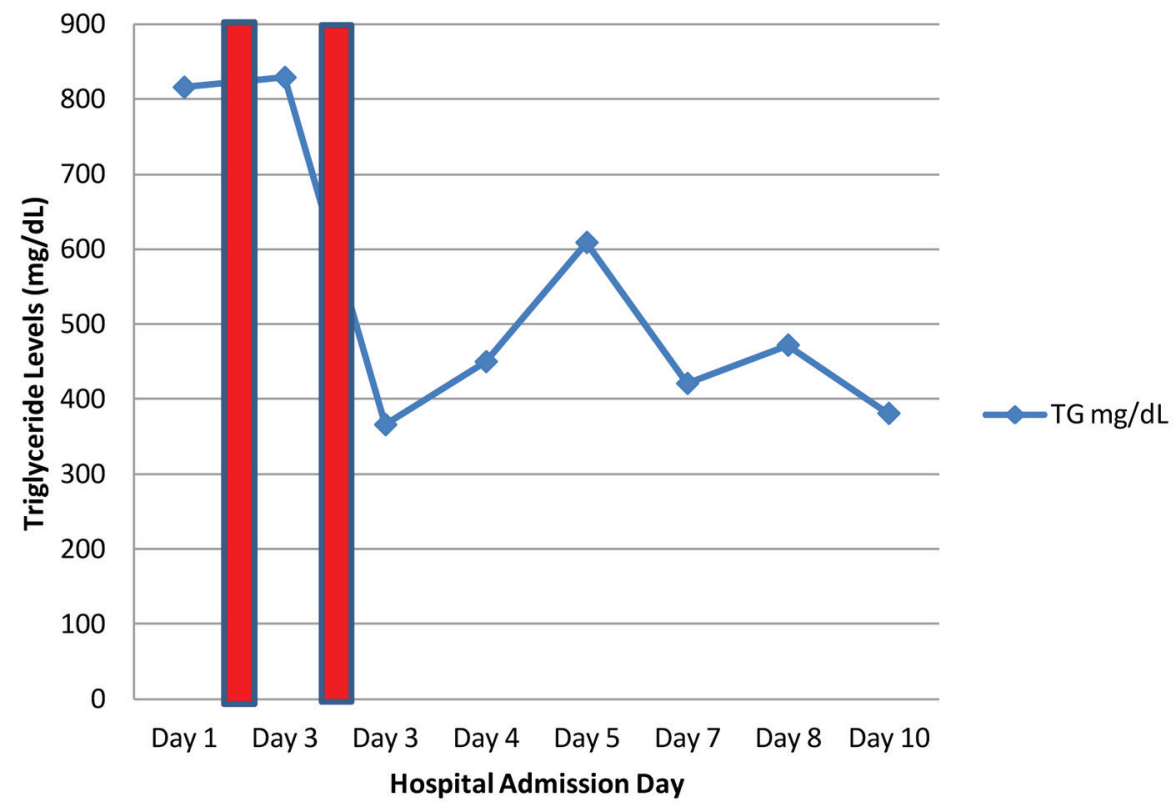

Figure 2. Triglyceride levels during hospital stay. The exact day 1 triglyceride level was undetermined but for graphical presentation, it has been included as the cutoff of $816 \mathrm{mg} / \mathrm{dL}$. Red bars represent plasmapheresis sessions. 
ment of a mass consistent with a pancreatic pseudocyst or abscess. The patient was observed and managed conservatively from this point forward. She was discharged home 12 days after admission on gemfibrozil and glyburide as maintenance therapy.

\section{Discussion}

The etiology of SHTG is not fully understood. SHTG may develop as a result of disturbances in the production and clearance of plasma lipoproteins in the setting of diabetes mellitus. It may also serve as a precursor to the development of diabetes mellitus [11]. The association of SHTG and acute pancreatitis was first postulated by Speck et al in 1865 and its underlying mechanisms include chylomicron-related, hyperviscosityinduced ischemia, free fatty acid-induced inflammation, and an underlying genetic predisposition.

Poorly controlled diabetes is the most common cause of hypertriglyceridemia, followed by alcoholism. The third most common causes are diet and medications, which are responsible for $15-20 \%$ of cases of hypertriglyceridemia. In the present case, the presence of SHTG was likely due to poorly controlled diabetes mellitus given the past medical history of gestational diabetes. Previous literature has documented a high prevalence of diabetes in SHAP $[5,12]$. As mentioned before, very high triglyceride levels $(>1,000 \mathrm{mg} / \mathrm{dL})$ are associated with SHAP, and these levels are rarely seen in the absence of other conditions. Yadav and Pitchumoni suggest that acute pancreatitis due to secondary hypertriglyceridemia alone is unlikely, making a multifactorial etiology probable [13]. Though genetic data were not available for our patient, we speculate that a combination of primary genetic influences as well as secondary causes of hypertriglyceridemia coexisted to result in this clinical presentation. However, hypertriglyceridemia secondary to diabetic ketoacidosis was the only cause of acute pancreatitis in the majority of patients recently reported by Fulop and Eder [14]. Indeed, no predisposing etiology other than hypertriglyceridemia due to poorly controlled diabetes was discovered in previous case reports of a similar triad of hypertriglyceridemia, diabetic ketoacidosis, and acute pancreatitis $[15,16]$.

Our patient had normal amylase and increased lipase levels at presentation, which supports the previous literature describing lipase as a more sensitive and specific marker of pancreatitis compared to amylase [17]. Moreover, lipemia-related interference could have underestimated amylase levels due to colorimetric amylase assay or the presence of plasma assay inhibitor and an increased non-aqueous phase of the specimen $[5,15]$.

As seen in the management of our patient, hypertriglyceridemia is usually addressed by pharmacotherapy with fibrates. Fibrates typically lower triglyceride levels rapidly and effectively and serve as first-line therapy for elevated triglycerides [18-20]. However, in the present case, gemfibrozil therapy failed to reduce triglyceride levels within the first $24 \mathrm{~h}$ after treatment, which necessitated an alternative therapeutic strategy. Thus, our decision to initiate therapeutic plasmapheresis was driven by the lack of improvement in the patient's clinical status with conservative management.

Plasmapheresis therapy employs the removal and replacement of large volumes of plasma in order to filter out pathologic antibodies, cytokines, immune complexes, or other blood components [21]. Two methods of plasmapheresis are currently available, centrifugation and filtration. Centrifugation relies on different densities of blood and plasma components which separate when centrifuged, allowing for removal of a particular layer, and thus, particular component of blood or plasma. Filtration, on the other hand, employs filters of varying sizes to separate plasma from larger components of blood including red blood cells, WBCs and platelets [22]. Hypertriglyceridemic pancreatitis is an indication for therapeutic plasmapheresis according to the 2010 Guidelines of the American Society for Apheresis, but is only given a category III indication, which denotes a condition for which the "optimum role of apheresis therapy is not established and decision making should be individualized" [23].

On average, the drop in triglyceride levels after one to three sessions of therapeutic plasmapheresis is between $46 \%$ and $80 \%$ [23]. A single session of plasmapheresis may rapidly reduce triglyceride levels by up to $70 \%[24,25]$. We were not able to calculate the percentage drop in triglyceride levels from baseline because the exact baseline triglyceride level was not determined by our laboratory (reported as $>816 \mathrm{mg} / \mathrm{dL}$ ). However, there was a 54\% drop in triglyceride levels after the second session of plasmapheresis. This rapid removal of lipid particles and triglyceridemia-associated pro-inflammatory agents is the main advantage of plasmapheresis over conservative management and has been effective in clinical practice. It is important to note that the maximal reduction in morbidity and mortality is evident when therapeutic plasmapheresis is used as early as possible $[26,27]$. This may explain the lack of benefit on the overall mortality and complications that was observed in the retrospective comparison of SHAP before and after the availability of plasmapheresis [28].

In our experience, plasmapheresis is a viable and effective therapy for adult SHAP and warrants further studies and perhaps, revision of its category III indication. Betteridge et al described the first case of SHAP that was successfully treated with therapeutic plasmapheresis in 1978 [29]. Several subsequent case reports have highlighted the role of apheresis in acute pancreatitis with SHTG [30-32]. Therapeutic plasmapheresis is also effective in treating SHAP of various etiologies, including drug therapy with isotretinoin, ritonavir, cyclosporine, asparaginase, as well as lipid-emulsion overdose with TPN therapy [23]. In two case-series reports, subsequent episodes in patients with recurring pancreatitis were reduced after ongoing therapeutic plasmapheresis, by as much as $67 \%$ $[24,33]$. Our case report confirms the efficacy of therapeutic plasmapheresis in the setting of acute pancreatitis and adds to the existing literature by presenting a case of SHTG and acute pancreatitis with a background of gestational diabetes, rather than long-standing diabetes. It is important for clinicians to be aware that patients may make their initial presentation with diabetes mellitus in this manner, and therefore, physicians should maintain a high-index of suspicion in patients with significant risk factors. 


\section{Conclusion}

This is the first case report regarding the use of therapeutic plasmapheresis in an adult with an initial presentation of diabetes mellitus with diabetic ketoacidosis complicated by acute pancreatitis and SHTG. Therapeutic plasmapheresis has been shown to be a safe and effective therapeutic option in the management of potentially life-threatening SHAP. Further experience is needed to solidify clinical evidence, study the effect on mortality and acute pancreatitis-related complications, and optimize future management guidelines for patients with SHTGinduced acute pancreatitis.

\section{Disclosures}

The authors report no conflicts of interest.

\section{References}

1. Osman MO, Jensen SL. Acute pancreatitis: the pathophysiological role of cytokines and integrins. New trends for treatment? Dig Surg. 1999;16(5):347-362.

2. Armstrong CP, Taylor TV. Pancreatic-duct reflux and acute gallstone pancreatitis. Ann Surg. 1986;204(1):5964.

3. Widdison AL, Karanjia ND. Pancreatic infection complicating acute pancreatitis. Br J Surg. 1993;80(2):148-154.

4. Yang AL, Vadhavkar S, Singh G, Omary MB. Epidemiology of alcohol-related liver and pancreatic disease in the United States. Arch Intern Med. 2008;168(6):649-656.

5. Fortson MR, Freedman SN, Webster PD, 3rd. Clinical assessment of hyperlipidemic pancreatitis. Am J Gastroenterol. 1995;90(12):2134-2139.

6. Toskes PP. Hyperlipidemic pancreatitis. Gastroenterol Clin North Am. 1990;19(4):783-791.

7. Anderson F, Thomson SR, Clarke DL, Buccimazza I. Dyslipidaemic pancreatitis clinical assessment and analysis of disease severity and outcomes. Pancreatology. 2009;9(3):252-257.

8. Roberts SE, Akbari A, Thorne K, Atkinson M, Evans PA. The incidence of acute pancreatitis: impact of social deprivation, alcohol consumption, seasonal and demographic factors. Aliment Pharmacol Ther. 2013;38(5):539-548.

9. Sandhu S, Al-Sarraf A, Taraboanta C, Frohlich J, Francis GA. Incidence of pancreatitis, secondary causes, and treatment of patients referred to a specialty lipid clinic with severe hypertriglyceridemia: a retrospective cohort study. Lipids Health Dis. 2011;10:157.

10. Tenner S, Baillie J, DeWitt J, Vege SS. American College of Gastroenterology guideline: management of acute pancreatitis. Am J Gastroenterol. 2013;108(9):1400-1415; 1416.

11. Kreisberg RA. Diabetic dyslipidemia. Am J Cardiol. 1998;82(12A):67U-73U; discussion 85U-86U.

12. Lloret Linares $C$, Pelletier AL, Czernichow S, Vergnaud AC, Bonnefont-Rousselot D, Levy P, Ruszniewski P, et al.
Acute pancreatitis in a cohort of 129 patients referred for severe hypertriglyceridemia. Pancreas. 2008;37(1):13-2.

13. Yadav D, Pitchumoni CS. Issues in hyperlipidemic pancreatitis. J Clin Gastroenterol. 2003;36(1):54-62.

14. Fulop M, Eder H. Severe hypertriglyceridemia in diabetic ketosis. Am J Med Sci. 1990;300(6):361-365.

15. Lutfi R, Huang J, Wong HR. Plasmapheresis to treat hypertriglyceridemia in a child with diabetic ketoacidosis and pancreatitis. Pediatrics. 2012;129(1):e195-198.

16. Winter RJ, Herr TJ, Stone NJ, Traisman HS. Diabetic lipemia in childhood diabetic ketoacidosis: a clue to coexisting acute pancreatitis. Diabetes Care. 1980;3(6):706707.

17. Treacy J, Williams A, Bais R, Willson K, Worthley C, Reece J, Bessell J, et al. Evaluation of amylase and lipase in the diagnosis of acute pancreatitis. ANZ J Surg. 2001;71(10):577-582.

18. Yuan G, Al-Shali KZ, Hegele RA. Hypertriglyceridemia: its etiology, effects and treatment. CMAJ. 2007;176(8):1113-1120.

19. McPherson R, Frohlich J, Fodor G, Genest J, Canadian Cardiovascular S. Canadian Cardiovascular Society position statement--recommendations for the diagnosis and treatment of dyslipidemia and prevention of cardiovascular disease. Can J Cardiol. 2006;22(11):913-927.

20. Barter PJ, Rye KA. Cardioprotective properties of fibrates: which fibrate, which patients, what mechanism? Circulation. 2006;113(12):1553-1555.

21. Winters JL. Plasma exchange: concepts, mechanisms, and an overview of the American Society for Apheresis guidelines. Hematology Am Soc Hematol Educ Program. 2012;2012:7-12.

22. Nguyen TC, Kiss JE, Goldman JR, Carcillo JA. The role of plasmapheresis in critical illness. Crit Care Clin. 2012;28(3):453-468, vii.

23. Szczepiorkowski ZM, Winters JL, Bandarenko N, Kim $\mathrm{HC}$, Linenberger ML, Marques MB, Sarode R, et al. Guidelines on the use of therapeutic apheresis in clinical practice--evidence-based approach from the Apheresis Applications Committee of the American Society for Apheresis. J Clin Apher. 2010;25(3):83-177.

24. Yeh JH, Chen JH, Chiu HC. Plasmapheresis for hyperlipidemic pancreatitis. J Clin Apher. 2003;18(4):181-185.

25. Lennertz A, Parhofer KG, Samtleben W, Bosch T. Therapeutic plasma exchange in patients with chylomicronemia syndrome complicated by acute pancreatitis. Ther Apher. 1999;3(3):227-233.

26. Ohmoto K, Neishi Y, Miyake I, Yamamoto S. Severe acute pancreatitis associated with hyperlipidemia: report of two cases and review of the literature in Japan. Hepatogastroenterology. 1999;46(29):2986-2990.

27. Furuya T, Komatsu M, Takahashi K, Hashimoto N, Hashizume T, Wajima N, Kubota M, et al. Plasma exchange for hypertriglyceridemic acute necrotizing pancreatitis: report of two cases. Ther Apher. 2002;6(6):454-458.

28. Chen JH, Yeh JH, Lai HW, Liao CS. Therapeutic plasma exchange in patients with hyperlipidemic pancreatitis. World J Gastroenterol. 2004;10(15):2272-2274.

29. Betteridge DJ, Bakowski M, Taylor KG, Reckless JP, 
de Silva SR, Galton DJ. Treatment of severe diabetic hypertriglyceridaemia by plasma exchange. Lancet. 1978;1(8078):1368.

30. Abel T, Sandor K, Tremmel A, Penzes I, Gamal EM, Lengyel G, Szabolcs I. [Plasmapheresis in the treatment of hypertriglyceridemia]. Orv Hetil. 2014;155(30):12031206.

31. Alkimawi K. Abdominal pain in hypertriglyceridemia.
Gastroenterol Rep (Oxf). 2014;2(3):237-238.

32. Iskandar SB, Olive KE. Plasmapheresis as an adjuvant therapy for hypertriglyceridemia-induced pancreatitis. Am J Med Sci. 2004;328(5):290-294.

33. Yeh JH, Lee MF, Chiu HC. Plasmapheresis for severe lipemia: comparison of serum-lipid clearance rates for the plasma-exchange and double-filtration variants. J Clin Apher. 2003;18(1):32-36. 